\title{
Semi-automated Speaker Adaptation: How to Control the Quality of Adaptation?
}

\author{
Andrey V. Savchenko \\ National Research University Higher School of Economics, Nizhniy Novgorod, \\ Russian Federation \\ avsavchenko@hse.ru
}

\begin{abstract}
Since the early 1990s, speaker adaptation have become one of the intensive areas in speech recognition. State-of-the-art batch-mode adaptation algorithms assume that speech of particular speaker contains enough information about the user's voice. In this article we propose to allow the user to manually verify if the adaptation is useful. Our procedure requires the speaker to pronounce syllables containing each vowel of particular language. The algorithm contains two steps looping through all syllables. At first, LPC analysis is performed for extracted vowel and the LPC coefficients are used to synthesize the new sound (with a fixed pitch period) and play it. If this synthesized sound is not perceived by the user as an original one then the syllable should be recorded again. At the second stage, speaker is asked to produce another syllable with the same vowel to automatically verify the stability of pronunciation. If two signals are closed (in terms of the Itakura-Saito divergence) then the sounds are marked as "good" for adaptation. Otherwise both steps are repeated. In the experiment we examine a problem of vowel recognition for Russian language in our voice control system which fuses two classifiers: the CMU Sphinx with speaker-independent acoustic model and Euclidean comparison of MFCC features of model vowel and input signal frames. Our results support the statement that the proposed approach provides better accuracy and reliability in comparison with traditional MAP/MLLR techniques implemented in the CMU Sphinx.
\end{abstract}

Keywords: Automatic speech recognition, phoneme recognition, speaker adaptation, CMU Sphinx, voice control, linear autoregression model.

\section{Introduction}

Nowadays the speaker adaptation is known to be the most efficient way to improve the automatic speech recognition (ASR) quality [1]. It allows to use relatively small amount (5-10 minutes) of speech data from the new user to transform the general speaker-independent (SD) acoustic model into speakerdependent (SD) one. In this article we explore practically important unsupervised batch-mode (static) adaptation in which the user has to pronounce several phrases/sounds before exploitation of the ASR system. Various adaptation techniques has been developed since 1990s when the HMM-GMM (Hidden Markov

A. Elmoataz et al. (Eds.): ICISP 2014, LNCS 8509, pp. 638-646, 2014.

(C) Springer International Publishing Switzerland 2014 
Model-Gaussian Mixture Model) approach became the state-of-the art in ASR [1, 2]. Traditional algorithms (MAP (Maximum A Posteriori) and MLLR (Maximum Likelihood Linear Regression)) modify the HMM parameters (usually, GMM's means) of the SI acoustic model to make the model closer match the particular speaker [1]. These algorithms (especially, MAP) usually require too many speech data to perform successful adaptation. Hence, the setup of the SD mode may require much time (up to 30 minutes). To prevent this drawback, other approaches such as vocal tract length normalization (VTLN) [3], clusterbased techniques (e.g., having distinct female and male acoustic model) [4] or eigenvoices (in which only four letters must be pronounced once by the user [2]) have recently appeared.

Unfortunately, none of these approaches allows the user to verify if his training data is enough to improve the ASR performance. Actually, the user should perform adaptation, test the recognition quality and repeat the adaptation if the accuracy is not satisfactory. No one can guarantee that the first phase is successful. That's why most commercial ASR products are mainly focused on SI mode.

In this article we investigate the possibility to improve the quality of adaptation procedure implemented in our voice control system 5 which requires the user to pronounce once each vowel of Russian language. We propose two tests. First, manual, test performs the simple speech synthesis on the basis of the linear autoregression model [6] and the LPC (linear prediction coding) coefficients evaluated from the user's vowel. If the synthesized signal sounds similarly to the original vowel from the user's point of view, then the second test is verified. Namely, the user pronounces the same vowel again and two produced signals are compared with the Itakura-Saito divergence. If the distance does not exceed a fixed threshold, the vowel is added to the speaker phonetic database. After completion of this procedure for all vowels, the recognition is done by simple aggregation of the outputs (posterior probabilities) of two ASR algorithms. The first one is any conventional ASR software (we use the CMU Sphinx [7]). The second algorithm involves the syllables extraction, phoneme segmentation and the recognition of a vowel in a syllable by comparison of all frames with each vowel from the speaker database (e.g., looking for the nearest neighbor) [5].

The rest of the paper is organized as follows: Section 2 introduces our speech signal synthesis and gives details of our recognition procedure. In Section 3, we present the experimental results in vowel phoneme recognition task. To show how our approach may be applied in practice we also present preliminary results in voice commands recognition with the requirement of isolated syllable mode [5]. Finally, concluding comments are given in Section 4.

\section{Semi-automated Speaker Adaptation}

The phoneme recognition task is to assign a query utterance $\mathbf{x}$ to one of the phoneme from the phonetic alphabet. We assume it contains $R$ vowel phonemes, each of them may be specified by the HMM-GMM parameters, model signals, etc. 
Closed, poorly distinguished, phonemes are united into $C \leq R$ clusters, where $c(r) \in\{1, \ldots, C\}$ is the $r$-th phoneme's cluster number. At the first, preliminary, stage of the proposed approach (Fig. 1) the speaker adaptation is performed. The user is required to pronounce twice each of $R$ phonemes as a part of a syllable with leading short unvoiced sound (e.g., "s") so that it is easy to extract the phoneme by any segmentation algorithm [1]. LPC coefficients $\left\{a_{i, m}^{(r)}\right\}$, $m \in\{1, \ldots, p\}$ are estimated for extracted phonemes $\left\{\mathbf{x}_{i}^{(r)}\right\}, m \in\{1,2\}$ by the Levinson-Durbin algorithm with the Burg method [1, [6]. Here $p$ is the LPC order. The next, verification step, is the key part of the adaptation. We use the known equivalence of the LPC analysis and linear autoregression (AR) model [6] and synthesize the new signal $\mathbf{y}^{(r)}$ (with initialization $y_{j}^{(r)}=0, j \in\{1, \ldots, p\}$ ):

$$
y_{j}^{(r)}=e_{j}-\sum_{m=1}^{p} a_{i, m}^{(r)} \cdot y_{p+j-m}^{(r)},
$$

where $e_{j}=\delta\left(j-f_{0} \cdot\left\lfloor\frac{j}{f_{0}}\right\rfloor\right), \delta(\cdot)$ and $\lfloor\cdot\rfloor$ are the discrete Dirac delta and floor functions, respectively, $f_{0}=$ const is the fundamental frequency of the synthesized speech. The signal $\mathbf{y}^{(r)}$ is played (produced) to the user who is required to manually verify the closeness of this signal and the pronounced phoneme. Unfortunately, this step cannot be automated due to the equivalence of LPC coefficients for signals $\mathbf{x}_{1}^{(r)}$ and $\mathbf{y}^{(r)}$. If signal $\mathbf{x}_{1}^{(r)}$ is verified and the ItakuraSaito (IS) distance between signals $\mathbf{x}_{1}^{(r)}$ and $\mathbf{x}_{2}^{(r)}$ is less than the fixed threshold $\rho_{0}=$ const (i.e., the phoneme pronunciation is rather stable), then the first model is added to the user phonetic database $\mathbf{x}_{r}^{*}=\mathbf{x}_{1}^{(r)}$. Otherwise the adaptation for the $r$-th phoneme is repeated. We apply the widely used in the ASR task IS distance as it is strongly correlated with subjective estimates of speech signals closeness, namely, MOS (mean opinion score) [1].

After completion of adaptation for all $R$ phonemes the phoneme recognition is performed. We propose to apply the classifier fusion (Fig. 1) to unite any ASR (in SI or SD mode if the fast adaptation on the basis of recorded phonemes is available) and the SD phoneme recognition with the speaker phonetic database $\left\{\mathbf{x}_{r}^{*}\right\}$ [5]. The former classifier is assumed to return the set of posterior probabilities $P_{A S R}(r \mid \mathbf{x})$. The latter one is based on comparison of conventional MFCC (Mel-frequency cepstral coefficients) features. Query utterance $\mathbf{x}$ is divided into overlapping frames $\mathbf{x}(t), t \in 1, \ldots, T$. Each $t$-th frame is put in correspondence with the closest phoneme $\mathbf{x}_{\nu(t)}^{*}$ (in terms of Euclidean distance with MFCC). Next, our phonetic coding method [8] is used to associate $\mathrm{x}$ with the fuzzy set $\{c, \mu(c)\}$ [9], where grade of membership

$$
\mu(c)=\sum_{t=1}^{T} \delta(c(\nu(t))-c) .
$$




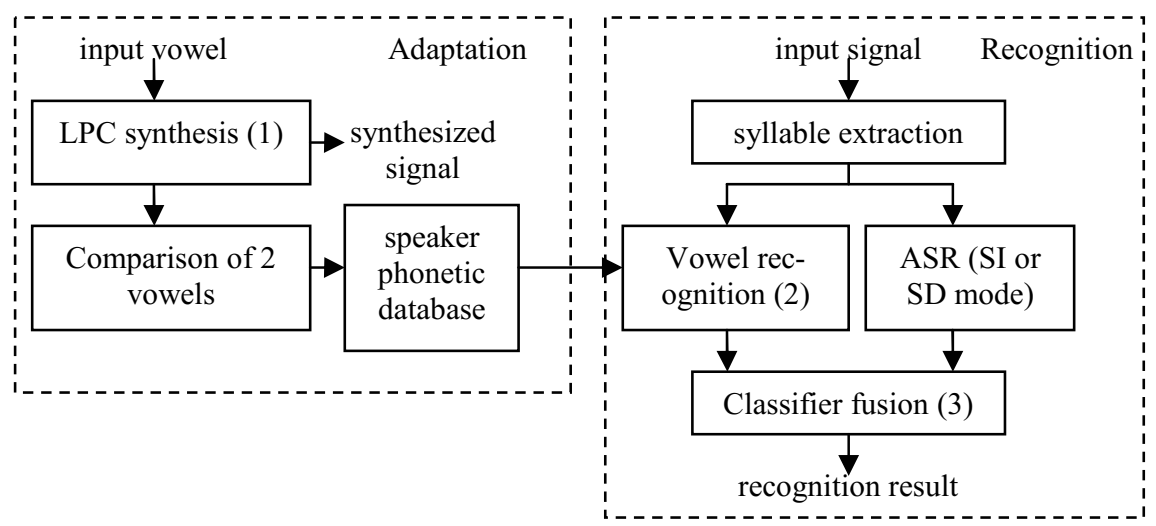

Fig. 1. Proposed phoneme recognition method with semi-automated speaker adaptation

Finally, the classifier results $P_{A S R}(r \mid \mathbf{x})$ and $\mu(c)$ are simply aggregated

$$
\mu_{\alpha}(c)=\alpha \cdot \sum_{r=1}^{R} \delta(c(r)-r) \cdot P_{A S R}(r \mid \mathbf{x})+(1-\alpha) \cdot \mu(c),
$$

where $0 \leq \alpha<1$ is the aggregation weight. The decision is made in favor of the phoneme code with the maximum grade $\mu_{\alpha}(c)$. The proposed approach to phoneme recognition (1)-(3) saves all advantages of the conventional ASR techniques but allows to increase the accuracy by performing fast robust speaker adaptation. The next section provides an experimental evidence to support this claim.

\section{Experimental Results}

In this section we examine the effectiveness of our approach in the task of Russian vowels recognition in the syllable. The noise-canceling microphone A4Tech HS-12o was used to record speech in the following format: PCM wav, mono, sampling rate $8000 \mathrm{~Hz}, 16$ bits per sample. The signal-noise ratio (SNR) is approximately equal to $30 \mathrm{~dB}$. To simplify the experiment, we require the speaker to pronounce words/phrases in isolated syllable mode. In such case, the mapping of the phrase's textual representation to its phonetic transcription becomes straightforward as all vowels are stressed [5]. The ASR quality was tested with 2 vocabularies (in Russian): a) the list of 1913 drugs solved in one pharmacy of Nizhny Novgorod (Russia) (hereinafter "Pharmacy"); b) the list of 1830 Russian cities with the corresponding regions, e.g., "Kstovo (Nizhegorodskaya)" (hereinafter "Cities"). All vocabularies are available in text files, each line contains separate word/phrase. Ten speakers (five men and five women of different age) pronounced each word from these vocabularies twice. The proposed algorithm of classifier fusion was implemented as a part of our voice control system [5]. The following parameters were chosen: frame length $30 \mathrm{~ms}$, frame overlap 10 
ms, LPC-model order $p=12$, the aggregation weight $\alpha=0.6$. An adaptive Wiener filter from the CMU Sphinx 4's source code was applied to reduce the noise [1], 7. At the preliminary stage, the phonetic database was adapted for each speaker. In configuration mode, the speaker clearly spoke 10 vowels of the Russian language and the pauses are removed. Two phonetic databases were created containing: 1) first attempt of vowel pronunciation ("no verification"), and 2) sounds verified by the proposed approach ("verification"). In the latter case, sound recording was repeated until the sound of the synthesized AR-process was close (by the speaker perception) to the pronounced vowel and the IS divergence between two repeated signals (for each vowel) is less than $\rho_{0}=0.6$. In average, every sound needed 2-3 iterations. Hence, the average time of phonetic database adaptation for a particular speaker took $2.75 \mathrm{~min}$. (minimal and maximal time is $1.25 \mathrm{~min}$. and $6.5 \mathrm{~min}$., respectively).

In our experiments we compare the recognition accuracy of the proposed approach with conventional CMU Sphinx. The latter was tested in both SI and SD modes. For SD mode, each speaker produced several times all vowels and 10 Russian phrases from the Voxforge 1 which are commonly used to adapt the acoustic model. Both MAP and MLLR adaptation was done and the best one (in terms of further accuracy) was chosen for each speaker.

In the first experiment syllables in each word were extracted by simple amplitude detector (pause was defined as the signal with low amplitude and duration exceeding $70 \mathrm{~ms}$.) and put in correspondence with one of $R=10$ Russian vowels. Hence, each vowel has been associated with different number of syllables (the more frequent is the vowel in the language, the more number of syllables is associated with it). According to Russian phonetics, vowels "a" ("aa" in Sphinx transcription) and "я" ("ja"), "y" ("uu") and "ю" ("ju"), "o" ("оo") and "ë" ("jo"), "э" ("еe") and "e" ("је"), "ы" ("уу") and "и" ("ii") were united into $C=5$ clusters. Hence, if the syllable containing one vowel of each cluster (e.g., "ja") was recognized as the other vowel in the same cluster (e.g., "a"), then the recognition result is assumed to be correct. The average error rate is presented in Table 1. As the number of recorded syllables is not equal for different vowels, we use here either micro averaging (total average error rate) or macro averaging (mean of error rates for all classes).

Though the test set size is rather large, the standard deviation of the error rate is not close to zero as different speakers are characterized by different error rate. It is not surprising that SD mode leads to an improvement of recognition accuracy in comparison with default SI mode. It is remarkable that the fusion with the vowel recognition with the user's phonetic database leads to better results than for conventional SD mode. And the most remarkable fact is the accuracy (especially, its standard deviation) increase if the phonetic database is verified by the user. It seems that such short training allows the speaker to understand better the requirement of the ASR system in stable pronunciation. To explore our results deeper, the average error rate for each vowel is shown in box-plots in Fig. 2.

${ }^{1}$ http://www .voxforge.org/ru/read 
Table 1. The average error rate (\%) for vowel phoneme recognition

\begin{tabular}{|l|c|c|c|c|}
\hline \multirow{2}{*}{ Averaging } & \multicolumn{2}{|c|}{ CMU Sphinx only } & \multicolumn{2}{c|}{ Fusion of CMU Sphinx with user vowel recognition } \\
\cline { 2 - 5 } & SI & SD & No verification & Verification \\
\hline Micro & $8.1 \% \pm 2.5 \%$ & $6.5 \% \pm 2.2 \%$ & $6.3 \% \pm 2.1 \%$ & $\mathbf{4 . 4 \%} \pm \mathbf{2 . 2 \%}$ \\
\hline Macro & $8.8 \% \pm 2.7 \%$ & $6.7 \% \pm 1.8 \%$ & $6.2 \% \pm 2.3 \%$ & $\mathbf{4 . 1 \%} \pm \mathbf{2 . 1 \%}$ \\
\hline
\end{tabular}

a

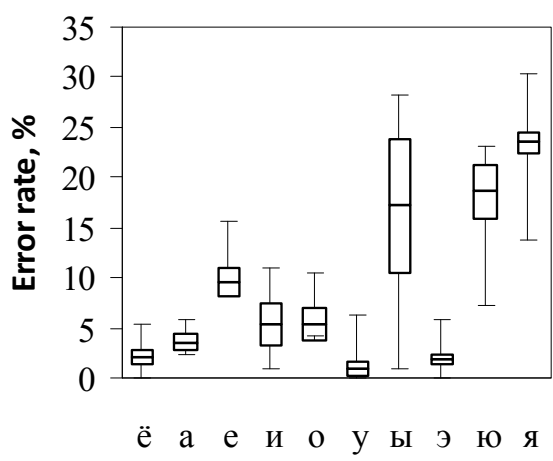

Vowel

C

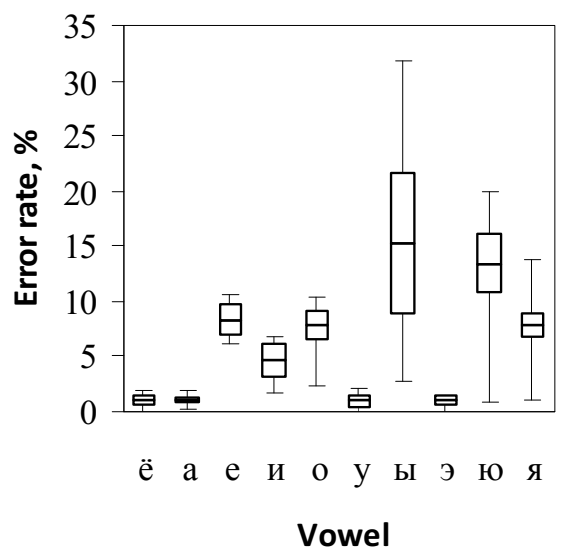

b

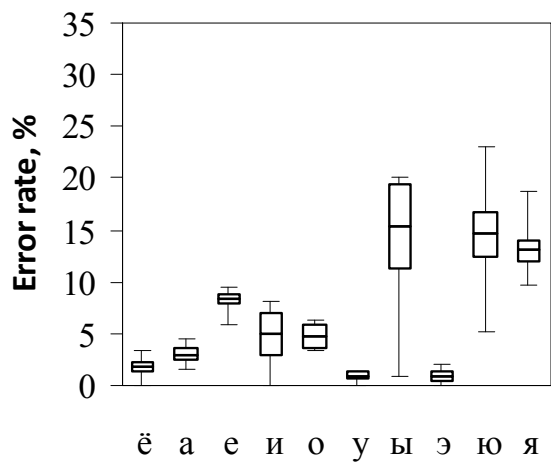

Vowel

$d$

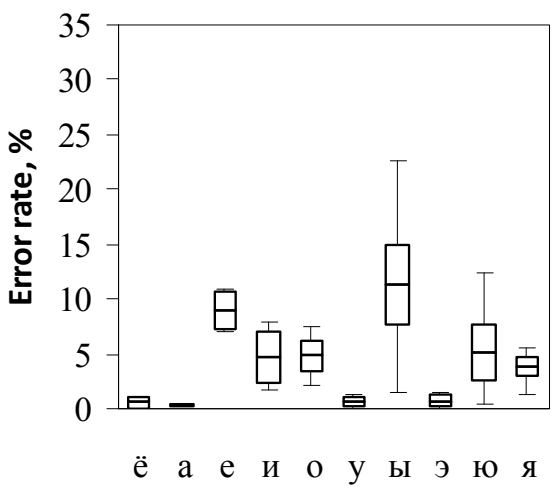

Vowel

Fig. 2. Dependence of average error rate (in \%) on the vowel class. a. CMU Sphinx with SI mode; b. CMU Sphinx with SD mode; c. The proposed algorithm (1)-(3) with no verification of the phonetic database ("first attempt"); d. Semi-automated verification.

As one can notice from comparison of these box-plots, our semi-automated verification allows to decrease either the mean of the error rate or its standard deviation. Our approach with classifier fusion (3) allows to get better results for 
several vowels (e.g., see the last box for "я"). The worst recognized vowel is "b" ("yy") which usually sounds quite different in distinct contexts.

In the last experiment we explore the isolated words recognition task for Russian language from the mentioned vocabularies. We again require the isolated syllable pronunciation to focus on vowel recognition in a context. The phonetic databases from the first experiment were used. The vowel phonemes were recognized in each syllable with the procedure described above (see the first experiment). Finally, each word is associated with the mean of grades $\mu_{\alpha}(c)$ of each syllable contained in this word. The word with the highest mean of grades is put into solution. If there is a set of several words which grades of memberships are equal, the recognition is assumed to be correct if the true word is contained in this set. We added an artificially generated white noise to each test signal (with SNR $30 \mathrm{~dB}, 20 \mathrm{~dB}, 10 \mathrm{~dB}$ ). The error rates for all described methods are summarized in Table 2 for the Pharmacy vocabulary and in Table 3 for the Cities vocabulary. Here we compare the algorithm of fusion of Sphinx and user vowel recognition not only with SI and SD modes of Sphinx, but also with vowel recognition (2) on the basis of the speaker phonetic database only (the second fused classifier) originally implemented in our voice control system [5].

According to these results we could draw the following conclusions. First, SD mode is a universal way to improve the ASR performance, though we should mentioned about several attempts of the speaker to repeat the training to achieve better testing results. Second, though vowel recognition with the speaker phonetic database [5] is characterized by low error rate (the best for the Pharmacy vocabulary, Table 2), it works fine only for small noise level. Third, the fusion of SD mode with the user vowel recognition (3) is the best choice in our experiment with isolated syllables. In this case the recognition error rate is $2.5 \%-8.6 \%$ lower than error rates of the SD mode. Finally, we demonstrated that the semi-

Table 2. The average error rate (\%), Pharmacy vocabulary

\begin{tabular}{|c|c|c|c|c|c|c|}
\hline \multirow{2}{*}{$\begin{array}{c}\text { SNR, } \\
\mathrm{dB}\end{array}$} & \multicolumn{2}{|c|}{ CMU Sphinx only } & \multicolumn{2}{c|}{ User phonemes only } & \multicolumn{2}{c|}{$\begin{array}{c}\text { Fusion of CMU Sphinx with } \\
\text { user vowel recognition }\end{array}$} \\
\cline { 2 - 7 } & SI & SD & First attempt & Verification & First attempt & Verification \\
\hline 30 & $13.7 \% \pm 8.5 \%$ & $8.8 \% \pm 6.4 \%$ & $8.1 \% \pm 3.6 \%$ & $\mathbf{3 . 7 \%} \pm \mathbf{0 . 9} \%$ & $6.3 \% \pm 1.2 \%$ & $5.0 \% \pm 0.6 \%$ \\
\hline 20 & $19.3 \% \pm 9.1 \%$ & $14.7 \% \pm 7.0 \%$ & $18.0 \% \pm 4.4 \%$ & $9.9 \% \pm 1.2 \%$ & $9.9 \% \pm 1.8 \%$ & $\mathbf{7 . 5 \%} \pm \mathbf{0 . 6 \%}$ \\
\hline 10 & $24.2 \% \pm 9.4 \%$ & $18.0 \% \pm 7.1 \%$ & $22.4 \% \pm 4.6 \%$ & $18.0 \% \pm 1.8 \%$ & $13.7 \% \pm 2.2 \%$ & $\mathbf{1 1 . 8 \%} \pm \mathbf{1 . 2 \%}$ \\
\hline
\end{tabular}

Table 3. The average error rate (\%), Cities vocabulary

\begin{tabular}{|c|c|c|c|c|c|c|}
\hline \multirow[t]{2}{*}{$\begin{array}{c}\mathrm{SNR}, \\
\mathrm{dB}\end{array}$} & \multicolumn{2}{|c|}{ CMU Sphinx only } & \multicolumn{2}{|c|}{ User phonemes only } & \multicolumn{2}{|c|}{$\begin{array}{c}\text { Fusion of CMU Sphinx with } \\
\text { user vowel recognition }\end{array}$} \\
\hline & SI & SD & First attempt & Verification & First attempt & Verification \\
\hline 30 & $9.2 \% \pm 7.5 \%$ & $7.8 \% \pm 5.7 \%$ & $11.4 \% \pm 5.2 \%$ & $4.4 \% \pm 1.2 \%$ & $2.6 \% \pm 1.2 \%$ & $0.9 \% \pm 0.4 \%$ \\
\hline 20 & $18.0 \% \pm 4.5 \%$ & $15.2 \% \pm 6.4 \%$ & $19.7 \% \pm 5.8 \%$ & $13.2 \% \pm 2.0 \%$ & $6.6 \% \pm 1.8 \%$ & $4.8 \% \pm 0.8 \%$ \\
\hline 10 & $20.6 \% \pm 4.5 \%$ & $15.6 \% \pm 6.5 \%$ & $25.0 \% \pm 6.0 \%$ & $22.8 \% \pm 2.5 \%$ & $11.8 \% \pm 2.2 \%$ & $9.6 \% \pm 1.0 \%$ \\
\hline
\end{tabular}


automated verification (Fig. 1) allows to choose better models and hence increase the accuracy at $1.3 \%-2.4 \%$ (for classifier fusion) and at $4.4 \%-8.1 \%$ for user vowel recognition without combining it with Sphinx.

\section{Conclusion}

In this paper we propose the ASR software to allow the user to verify the quality of speaker adaptation. Plenty of paper devoted to the improving adaptation with the con-temporary ASR techniques were presented. However, practically no one of them tries to estimate the "goodness" of training data. We offer here to use quite simple speech synthesis on the basis of the AR model (1) to present the user how his recording is perceived by the system. Unfortunately, it is impossible to perform verification in fully automatic mode as the GMM+MFCC model of synthesized signal is absolutely identical to the model of the signal recorded by the user. Comparison with SI acoustic model cannot help as it is not known if the recorded signal just contains some mistake in pronunciation of the speaker or it is a distinctive feature of the speaker's vocal tract. One key advantage of our approach is the possibility to apply it as a plug-in to any ASR system as it is demonstrated in our experiment (Tables 1-3) with the CMU Sphinx. The same vowel recordings may be used for state-of-the-art fast adaptation algorithms, e.g. eigenvoices. For now we demonstrated the potential of our approach in ASR with isolated syllable mode. Though this restriction is not so strict in Russian voice control applications, the most significant further direction of our semi-automated speaker adaptation is its application with continuous ASR.

Acknowledgement. This study was carried out within "The National Research University Higher School of Economics Academic Fund Program in 2013-2014, research grant No. 12-01-0003".

\section{References}

1. Benesty, J., Sondh, M., Huang, Y. (eds.): Springer Handbook of Speech Recognition. Springer (2008)

2. Kuhn, R., Junqua, J.C., Nguyen, P., Niedzielski, N.: Rapid speaker adaptation in eigenvoice space. IEEE Transactions on Speech and Audio Processing 8(6), 695-707 (2000)

3. Kim, D.Y., Umesh, S., Gales, M.J.F., Hain, T., Woodland, P.: Using VTLN for broadcast news transcription. In: ICSLP 2004(2004)

4. Yu, K., Gales, M.J.F.: Discriminative cluster adaptive training. IEEE Transactions on Speech and Audio Processing 14, 1694-1703 (2006)

5. Savchenko, A.V.: Phonetic words decoding software in the problem of Russian speech recognition. Automation and Remote Control 74(7), 1225-1232 (2013)

6. Marple Jr, S.L.: Digital Spectral Analysis: With Applications. Prentice-Hall Series in Signal Processing (1989)

7. CMU Sphinx, http://cmusphinx.sourceforge.net/ 
8. Savchenko, A.V.: Phonetic coding method in isolated words recognition problem. Journal of Communications Technology and Electronics 59(4), 310-315 (2014)

9. Savchenko, L.V., Savchenko, A.V.: Fuzzy Phonetic Decoding Method in a Phoneme Recognition Problem. In: Drugman, T., Dutoit, T. (eds.) NOLISP 2013. LNCS (LNAI), vol. 7911, pp. 176-183. Springer, Heidelberg (2013) 